\title{
BMJ Open Marijuana smoking and asthma: a protocol for a meta-analysis
}

\author{
Jincheng Lei, ${ }^{1}$ Mingjie Shao (1) ${ }^{2,3}$
}

To cite: Lei J, Shao M. Marijuana smoking and asthma: a protocol for a meta-analysis. BMJ Open 2022;12:e047324. doi:10.1136/ bmjopen-2020-047324

- Prepublication history for this paper is available online. To view these files, please visit the journal online (http://dx.doi org/10.1136/bmjopen-2020047324).

Received 25 November 2020 Accepted 01 November 2021

D) Check for updates

(c) Author(s) (or their employer(s)) 2022. Re-use permitted under CC BY-NC. No commercial re-use. See rights and permissions. Published by BMJ.

${ }^{1}$ Central South University Xiangya School of Public Health, Changsha, Hunan, China

${ }^{2}$ General Surgery

(Gastrointestinal Surgery), Xiangya Hospital Central South University, Changsha, China ${ }^{3}$ National Clinical Research Center for Geriatric Disorders, Xiangya Hospital Central South University, Changsha, China

Correspondence to

Mingjie Shao;

148302041@csu.edu.cn

\begin{abstract}
Introduction Recent studies have raised the concern on the risk of asthma in marijuana smokers; however, the results remain controversial and warrant further investigation. With a growing number of marijuana smokers, examining the association between marijuana smoking and asthma and quantifying such association through meta-analysis have important implications for public health and clinical decision-making. In view of this, the present protocol aims to detail a comprehensive plan of meta-analysis on the association aforementioned. The findings are expected to strengthen the current knowledge base pertaining to the potential adverse effects of marijuana smoking on pulmonary health and to facilitate the development of prevention strategies for asthma. Methods and analysis The MEDLINE/PubMed, Web of Science and EMBASE databases will be searched systematically from inception to 1 September 2021 to retrieve the relevant observational studies focusing on the association between marijuana smoking and asthma. Both unadjusted and adjusted effect sizes, such as 0R, relative risk, HR and the corresponding $95 \%$ Cls will be extracted for pooled analyses. Heterogeneity and publication bias across the included studies will be examined. The Newcastle-Ottawa Quality Scale will be used to assess the quality and risk of bias. Statistical software Review Manager V.5.3 and Stata V.11.0 will be used for statistical analyses.
\end{abstract}

Ethics and dissemination Since no private and confidential patient data will be included in the reporting, approval from an ethics committee is not required. The results will be published in a peer-reviewed journal or disseminated in the relevant conferences. The study raises no ethical issue.

OSF registration number 10.17605/OSF.IO/UPTXC.

\section{INTRODUCTION}

Marijuana is one of the most frequently administrated psychoactive drugs in the global context. ${ }^{1}$ As reported, the number of current marijuana smokers aged $\geq 12$ years in the USA had grown from $6.2 \%$ in 2002 to $10.1 \%$ in $2018 .^{2}$ In view of the potential benefits of marijuana observed in a range of medical conditions (eg, epilepsy, pain symptom, multiple sclerosis, anxiety and depression), 33 states in the USA have legalised the use of marijuana for medical purposes; additionally, the District of Columbia and another 11 states have legalised marijuana for recreational
Strengths and limitations of this study

- This is the first meta-analysis that aims to evaluate the association between marijuana smoking and asthma. Our findings are expected to strengthen the current knowledge base pertaining to the potential adverse effects of marijuana smoking on pulmonary health and to facilitate the development of prevention strategies for asthma.

- The study selection, data extraction and quality assessment will be performed by two independent investigators. Disagreements, if any, will be resolved by consulting a third investigator.

- The study quality and risk of bias will be comprehensively examined by adopting the NewcastleOttawa Quality Scale.

- Since this meta-analysis will include both prospective and retrospective studies, the final results may be subjected to bias to a certain extent. Meanwhile, the variations in study design and sample characteristics might result in a high level of heterogeneity.

- This meta-analysis intends to examine the associations between different frequencies of marijuana smoking and asthma as a subgroup analysis; however, it might be challenging or even impossible to implement due to the expected small number of qualified articles.

purposes. ${ }^{3-5}$ On the other hand, marijuana smoking is also suspected to be associated with several adverse effects such as the risk of chronic psychiatric disorders, cognitive decline, malignancy, chronic bronchitis, addiction and cardiovascular diseases. ${ }^{6}$

Cytologic variations in the respiratory tract, respiratory symptoms (eg, wheezing, cough, sputum production and shortness of breath) and impaired lung function are all potentially respiratory effects associated with marijuana smoking. ${ }^{7}$ Previous research suggested that marijuana smoking might expose users to carbon monoxide and tar deposition at a chance of 3-5 times higher than tobacco smokers, and the incidence of airflow obstruction caused by marijuana smoking was equivalent to 2.5-5 times of that caused by tobacco smoking. ${ }^{8-10}$ Since tobacco smoking is a recognised risk factor for the development of asthma, it has been speculated that marijuana 
smoking could also increase the risk of developing asthma. On the contrary, some other studies reported that marijuana smoking was not necessarily associated with asthma. ${ }^{11-15}$ A review concluded that even though a high frequency of marijuana smoking might lead to respiratory symptoms in the general population, the association between marijuana smoking and asthma should be further clarified. ${ }^{16}$ This association may be attributed to $\Delta$ 9-tetrahydrocannabinol (the physiologically active bronchodilator compound in marijuana), which has been demonstrated to cause rapid reversal of the bronchoconstriction induced experimentally by exercises or methacholine inhalation in asthma patients, ${ }^{17}$ and may have a therapeutic potential for asthma. ${ }^{18}$

Meta-analysis, as an important and useful tool to reveal the trends that are not apparently straightforward, can effectively facilitate researchers to establish clinical policies and guidelines. Therefore, we plan to perform a systematical meta-analysis on the existing literature to test the hypothesis that marijuana smoking is associated with a significantly greater risk of asthma.

\section{METHODS}

\section{Literature search}

This protocol was designed by referencing to the Preferred Reporting Items for Systematic Review and Meta-Analysis Protocols $2015,{ }^{19}$ and was registered in the Open Science Framework registries (osf.io/uptxc). A systematical search will be performed across MEDLINE/ PubMed, Web of Science and EMBASE from inception to 1 September 2021 to retrieve the relevant studies focusing on the association between marijuana smoking and asthma. Keywords or medical subject heading terms will be defined appropriately for the literature search (see table 1 for the detailed electronic search strategy), and necessary adaption will be made to all the search terms in order to cater the specific syntax rules of each database.

\section{Study selection}

Two investigators will be engaged independently to identify observational studies for inclusion according to the predetermined criteria. Disagreements, if any, shall

\begin{tabular}{ll}
\hline Table 1 & Electronic search strategy in PubMed \\
\hline Number & Search terms \\
\hline 1 & $\begin{array}{l}\text { marijuana(MeSH Terms) or marijuana(tiab) } \\
\text { or cannabis(tiab) or hemp(tiab) or } \\
\text { mariguana(tiab) or marihuana(tiab) or } \\
\text { dronabinol(tiab) or cannabichromene(tiab) or } \\
\text { tetrahydrocannabivarin(tiab) or nabilone(tiab) }\end{array}$ \\
2 & $\begin{array}{l}\text { asthma(MeSH Terms) or bronchial spasm(tiab) } \\
\text { or wheez*(tiab) or bronchospas?(tiab) } \\
\text { or bronchoconstrict?(tiab) or bronchial } \\
\text { hyperreactivity(tiab) or respiratory } \\
\text { hypersensitivity(tiab) }\end{array}$ \\
\hline 3 & 1 AND 2 \\
\hline
\end{tabular}

be resolved by consulting a third investigator as far as possible. Specifically, the inclusion criteria are: (1) observational studies including case-control studies, cohort studies and cross-sectional studies; (2) studies with at least one joint-year exposure (equivalent to one joint per day for 1 year) or more cumulative marijuana smoking (defined as ever smoking); (3) studies with clear diagnostic criteria for asthma (either clinically diagnosed or self-reported through interview; asthma-like disorders were not considered as asthma); (4) studies reporting the prevalence of asthma both in the groups of marijuana smokers and non-smokers and (5) studies published in English. Meeting abstracts, case reports, letters, reviews, cost-effective studies or non-human studies will not be included. In addition, studies setting marijuana use in the form of vaporisers as the study exposure will also be excluded, as this configuration is beyond the scope of the proposed meta-analysis.

\section{Data extraction}

The same two investigators will be responsible for extracting the following data independently using a standardised data collection form: publication information ((author(s) and year of publishing)); study information (country of origin, data sources, study setting and study period); demographics (age, sex distribution and sample size); exposure information (marijuana smoking) and outcome information (diagnosis of asthma). Then, both unadjusted and adjusted effect sizes, such as OR, relative risk (RR) or HR, will be extracted directly or, if not available, calculated based on the relevant data in the original studies. If such data for calculation cannot be found, the author(s) of the concerned study will be contacted directly to request for the missing information whenever possible. Any disagreements between the two investigators will be resolved by consulting a third investigator.

\section{Assessment of risk of bias}

The study quality will be examined by adopting the Newcastle-Ottawa Quality Scale. ${ }^{20}$ The risk of bias will be evaluated by the two investigators independently, and disagreements, if any, will be resolved by consulting a third investigator.

\section{Data analysis}

First, the study characteristics will be summarised with narrative texts and baseline tables, and the pooled OR, RR and HR as well as the corresponding 95\% CIs will be calculated. Both unadjusted and adjusted estimates will be pooled for analyses. Then, the heterogeneity across the included studies will be examined using Cochrane's $Q$ test and $\mathrm{I}^{2}$ statistics ( $p>0.05$ for $Q$ statistics and $\mathrm{I}^{2}<50 \%$ indicating statistical homogeneity). If statistical homogeneity is confirmed, the fixed-effects model will be applied, and otherwise, the random-effects model will be applied to pool the data. Statistical analyses will be performed using Stata V.11.0 and Review Manager V.5.3 (the Nordic Cochrane Centre, the Cochrane Collaboration, 
Copenhagen, 2014), with $\mathrm{p}<0.05$ indicating statistical significance. Finally, subgroup analyses will be performed (eg, age, sex, ethnic group, economic condition, education restrictions, geographical limitations, frequency of marijuana smoking) whenever feasible in order to minimise the impact of heterogeneity.

\section{Assessment of publication bias}

Publication bias will be examined if more than 10 studies are included by using the funnel plot and Egger test. Publication bias is confirmed if the funnel plot demonstrates an asymmetric pattern, and accordingly, the bias will be discussed and explained in detail.

\section{Patient and public involvement}

Given that this systematic review will be carried out based on published studies, patients and members of the public will not be involved directly. Only data from published literature and/or the aforementioned sources will be used.

\section{Ethics and dissemination}

This meta-analysis will be performed on published studies without involving any private and confidential patient data, so no approval is required from an ethics committee. The results will be reported by publishing in a peer-reviewed journal or disseminated in the relevant conferences. No ethical issues will be raised.

\section{DISCUSSION}

Asthma, a prevalent disease in children and adolescents, is deemed to be linked with significant health, social and financial issues. ${ }^{21}{ }^{22}$ With a growing number of marijuana smokers, especially in adolescents, ${ }^{2}$ examining the association between marijuana smoking and asthma and quantifying such association through meta-analysis have important implications for public health and clinical decision-making. To the best knowledge of the authors, at least 10 studies have examined the association between marijuana smoking and asthma, of which, five suggested that marijuana smoking was positively associated with asthma, ${ }^{1223-26}$ but such association was not supported by another five studies. ${ }^{11-14}$ Therefore, the association between marijuana smoking and asthma remains controversial, and the inconsistency among different studies may be attributed to the variations in study design and sample characteristics. It is noteworthy that the sample size of many previous studies might be too small to achieve a sufficiently strong statistical power, which explains, at least in part, why statistical difference was not reached in some instances despite an apparent trend. In view of the situation above, the proposed meta-analysis aims to summarise the available evidence to date to comprehensively examine the association between marijuana smoking and asthma. However, unlike medications that are usually standardised in their dose and composition, marijuana joints can be composed of different strains and chemicals. Thus, this study will review the strains or composition of marijuana plants used in the included studies and examine whether marijuana belongs to medical grade. The findings of this study are expected to strengthen the current knowledge base pertaining to the potential adverse effects of marijuana on pulmonary health and to facilitate the development of prevention strategies for asthma.

Contributors Conceptualisation: JL and MS. Data curation: JL and MS. Methodology: JL and MS. Writing of original draft: JL. Review and editing: MS.

Funding This work was supported by the National Natural Science Foundation of China (82000585), the Natural Science Foundation of Hunan Province, China(2021JJ41016).

Competing interests None declared.

Patient and public involvement Patients and/or the public were not involved in the design, or conduct, or reporting or dissemination plans of this research.

Patient consent for publication Not required.

Provenance and peer review Not commissioned; externally peer reviewed.

Open access This is an open access article distributed in accordance with the Creative Commons Attribution Non Commercial (CC BY-NC 4.0) license, which permits others to distribute, remix, adapt, build upon this work non-commercially, and license their derivative works on different terms, provided the original work is properly cited, appropriate credit is given, any changes made indicated, and the use is non-commercial. See: http://creativecommons.org/licenses/by-nc/4.0/.

ORCID iD

Mingjie Shao http://orcid.org/0000-0003-0965-7343

\section{REFERENCES}

1 Shah S, Patel S, Paulraj S, et al. Association of marijuana use and cardiovascular disease: a behavioral risk factor surveillance system data analysis of 133,706 us adults. Am J Med 2021;134:e1:614-20.

2 Key Substance Use and Mental Health Indicators in the United States. Results from the 2018 national survey on drug use and health. 2020. Substance Abuse and Mental Health Services Administration, 2019.

3 Daniller A. Two-thirds of Americans support marijuana legalization.. 2020. Pew Research Center, 2019.

4 Ladegard K, Thurstone C, Rylander M. Marijuana legalization and youth. Pediatrics 2020;145:S165-74.

5 Keyhani S, Steigerwald S, Ishida J, et al. Risks and benefits of marijuana use: a national survey of U.S. adults. Ann Intern Med 2018;169:282-90.

6 Volkow ND, Baler RD, Compton WM, et al. Adverse health effects of marijuana use. N Engl J Med Overseas Ed 2014;370:2219-27.

7 Owen KP, Sutter ME, Albertson TE. Marijuana: respiratory tract effects. Clin Rev Allergy Immunol 2014;46:65-81.

8 Coogan PF, Castro-Webb N, Yu J, et al. Active and passive smoking and the incidence of asthma in the black women's health study. Am J Respir Crit Care Med 2015;191:168-76.

9 Gilliland FD, Islam T, Berhane K, et al. Regular smoking and asthma incidence in adolescents. Am J Respir Crit Care Med 2006;174:1094-100.

10 Jaakkola MS, Piipari R, Jaakkola N, et al. Environmental tobacco smoke and adult-onset asthma: a population-based incident casecontrol study. Am J Public Health 2003;93:2055-60.

11 Schweitzer RJ, Wills TA, Tam E, et al. E-cigarette use and asthma in a multiethnic sample of adolescents. Prev Med 2017;105:226-31.

12 Wills TA, Choi K, Pagano I. E-Cigarette use associated with asthma independent of cigarette smoking and marijuana in a 2017 national sample of adolescents. J Adolesc Health 2020;67:524-30.

13 Larsen K, Faulkner GEJ, Boak A, et al. Looking beyond cigarettes: are Ontario adolescents with asthma less likely to smoke ecigarettes, marijuana, waterpipes or tobacco cigarettes? Respir Med 2016;120:10-15.

14 Han B, Gfroerer JC, Colliver JD. Associations between duration of illicit drug use and health conditions: results from the 20052007 national surveys on drug use and health. Ann Epidemiol 2010;20:289-97.

15 Bechtold J, Simpson T, White HR, et al. Chronic adolescent marijuana use as a risk factor for physical and mental health problems in young adult men. Psychol Addict Behav 2015;29:552-63. 
16 Self TH, Shah SP, March KL, et al. Asthma associated with the use of cocaine, heroin, and marijuana: a review of the evidence. $J$ Asthma 2017;54:714-22.

17 Tashkin DP, Shapiro BJ, Lee YE, et al. Effects of smoked marijuana in experimentally induced asthma. Am Rev Respir Dis 1975;112:377-86.

18 Tashkin DP. Airway effects of marijuana, cocaine, and other inhaled illicit agents. Curr Opin Pulm Med 2001;7:43-61.

19 Moher D, Shamseer L, Clarke M, et al. Preferred reporting items for systematic review and meta-analysis protocols (PRISMA-P) 2015 statement. Syst Rev 2015;4:1.

20 Stang A. Critical evaluation of the Newcastle-Ottawa scale for the assessment of the quality of nonrandomized studies in metaanalyses. Eur J Epidemiol 2010;25:603-5.

21 Akinbami LJ, Simon AE, Rossen LM. Changing trends in asthma prevalence among children. Pediatrics 2016;137:e20152354.
22 Kopel LS, Phipatanakul W, Gaffin JM. Social disadvantage and asthma control in children. Paediatr Respir Rev 2014;15:256-63.

23 Dai H, Richter KP. A national survey of marijuana use among US adults with medical conditions, 2016-2017. JAMA Netw Open 2019;2:e1911936.

24 Winhusen T, Theobald J, Kaelber DC, et al. Regular cannabis use, with and without tobacco co-use, is associated with respiratory disease. Drug Alcohol Depend 2019;204:107557.

25 Han Y-Y, Forno E, Celedón JC. Health risk behaviors, violence exposure, and current asthma among adolescents in the United States. Pediatr Pulmonol 2019;54:237-44.

26 Chabarria KC, Racusin DA, Antony KM, et al. Marijuana use and its effects in pregnancy. Am J Obstet Gynecol 2016;215:506.e1-506. e7. 